\title{
Analysis of Postural Stability During Continuous External Perturbations
}

\author{
Youngkyun Shin* ${ }^{*}$ Gu-Bum Park ${ }^{*}$
}

\begin{abstract}
The functional behaviors of human standing postural control were investigated when they were exposed to long-term horizontal vibration in the sagittal plane. For complexity of human postural control, a useful alternative method that has been based on a black-box approach was taken; that is, where the feedback mechanism was lumped into a single element. A motor-driven support platform was designed as a source of vibration. The AC Servo-controlled motors produced continuous anterior/posterior (AP) motion. The data were analyzed both in the time and frequency domain. The cross-correlation and coherency functions were estimated. Subjects behaved as a non-rigid pendulum with a mass and a spring throughout the whole period of the platform motion, as consistent with the plan chosen for this study.
\end{abstract}

Key Words : Standing Postural Control, Horizontal Vibration, Cross-correlation Analysis, Coherence Function

\section{Introduction}

Human posture dynamics has attracted many researchers in the field, and a number of studies have investigated standing postural control that have employed transient perturbation to evoke characteristics of postural responses [1-2]. Transient perturbation may be useful in

* Main author : Instructor, Department of Electrical Engineering, Yuhan University

** Corresponding author: Professor, Department of Electrical Engineering, Yuhan University

Tel : +82-2-2610-0734, Fax : +82-2-2619-9771

E-mail:pgb@yuhan.ac.kr

Date of submit : 3013. 3.27.

First assessment : 2013. 44.

Completion of assessment : 2013. 6.17. demonstration trigger specific and equally transient motor programs with respect to the standing posture mechanism. For instance, a sudden translation or a short acceleration demonstrated that the control mechanism is expressed by mainly reflexive responses. However, this may not be directly related to the continuous regulation of postural balance control. Hence, the use of continuously applied perturbation may be appropriate to study postural control behavior, which itself is a continuously maintaining process.

The inverted pendulum model is often used for standing posture study. When a standing body is exposed to anterior-posterior (AP) perturbation, the ankle joint firstly generates reactionary movement for balance control. Horak and Nashner [2-4] 
established a theoretical basis for ankle and hip strategies, McCllum and Leen [5] also showed that stiffening the body condition so that it acts as a one-segment or a two-segment inverted pendulum, such as with the ankle or the hip strategy, respectively.

Since the complexity of the biomechanics of the human standing posture dynamics, however, regarding the freestanding body as an inverted pendulum may oversimplify $[1,5]$ the mechanism of postural control, this may mislead understanding of postural control behavior. Hence, through the restriction of the subject standing body condition as a low degree of freedom, simplification of the system is essential for understanding the basis of the complexity of standing posture. Although many studies have investigated postural control in response to external disturbances, few reports exist to explicitly explain how subjects behave under such circumstances [6-10].

Three significant assumptions were adopted in this study. Firstly, the motion of standing posture complexity mainly occurs in the sagittal plane. Secondly, standing posture complexity only allows for flexion/extension motion, so that the translational motion of the standing posture complexity is assumed to be negligible. Thirdly, during AP platform motion, the subject's knee is tightly fixed, so that ankle and hip joint rotation were assumed to contribute to maintain body balance.

An AC Servo motor controlled vibrator was designed to apply a source of external vibration. Sinusoidal vibrations were used as input. Kinematic data were collected using a Vicon movement analysis system. Displacement and acceleration of the ankle, hip, and head were used as the output. Through a cross-correlation analysis, the measured data were first analyzed in the time domain. The coherence function was estimated over a frequency range (0.1-1
$\mathrm{Hz}$ ). The model was not purely based on inverted pendulum body dynamics, but rather on a three-link segment model on a movable support base.

\section{MATERIALS AND METHODS}

\subsection{Subject}

The physical characteristics of the four healthy subjects (mean age $\pm \mathrm{SD}, 27.5 \pm 5.2$ years) who participated in this study are shown in Table 1 . No subjects had any evidence or history of neurological, gate, postural or musculoskeletal impairments. All participants gave their informed consent prior to inclusion in the study. All subjects completed all trials without falling, stepping or reaching for a stable support. During the experiment, each subject was instructed to stand upright on the platform in a standardized stance; the feet of the subject were separated medio-laterally by a distance of about 10 $\mathrm{cm}$, and kept together so that the left and right ankle joints rotate about the same axis.

The subject's knee is tightly fixed during AP platform motion, so the ankle and hip joint rotation were assumed to contribute to maintaining body balance (Fig. 1). A number of medical appliances (fixing assembly) were used, including Velcro straps, medical splints and casts.

Table 1. Physical characteristics of the subjects (\# sign indicates the subject number)

\begin{tabular}{|c|c|c|c|}
\hline Subject & Age (yr) & Height $(\mathrm{m})$ & Weight $(\mathrm{kg})$ \\
\hline$\# 1$ & 23 & 1.70 & 65 \\
\hline$\# 2$ & 23 & 1.68 & 58 \\
\hline$\# 3$ & 32 & 1.74 & 64 \\
\hline$\# 4$ & 32 & 1.74 & 75 \\
\hline $\begin{array}{c}\text { Mean } \\
(\mathrm{SD})\end{array}$ & $27.5(5.2)$ & $1.72(0.03)$ & $65.5(7.05)$ \\
\hline
\end{tabular}




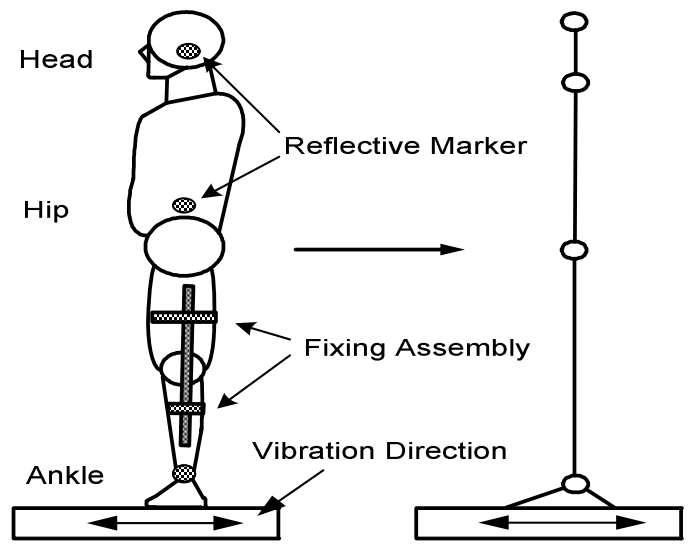

Fig. 1. The schematic of human standing posture exposed to horizontal vibration

After each trial, rest intervals were included to avoid subject exhaustion. The subjects stood barefoot with their arms folded comfortably across the chest and their head facing forward and upright. This arm position was adopted in order to eliminate the possibility of arm sway entering into the dynamics. The condition of the feet that were assumed not to slide or lift at the heel or toe, and since a triangular foot model is considered, it also did not enter into the dynamics of the system. Visual variations were added to the subjects' condition. The subjects' eyes were either open (EO) and fixed upon a target at eye level, or closed (EC) by blindfolding.

\subsection{Experimental setup}

The AC Servo-motor controlled vibrator was designed as a mobile rigid platform. The vibrator system consisted of an AC Servo-motor (Sanyo-Denki Co.) and actuator unit (THK Co.). It had a maximum stroke of $1200 \mathrm{~mm}$, maximum frequency of $5 \mathrm{~Hz}$, and maximum load of $100 \mathrm{Kgf}$. The platform was $606 \times 406 \mathrm{~mm}$ in size. Different sinusoidal vibrations were devised from $0.25 \mathrm{~Hz}$ to $1 \mathrm{~Hz}(0.25,0.5,0.75,1.0 \mathrm{~Hz})$. These input were devised for testing severe conditions that could generate levels near the limits of human upright standing postural control responses.

The duration of each trial was $40 \mathrm{~s}$. The initial period was not acquired in order to eliminate from the acquisition non-stationary events possibly induced by the onset of platform translation. Data from the latter $35 \mathrm{~s}$ were used in the subsequent analyses. Kinematic data were collected using a Vicon movement analysis system (Vicon 460, Oxford Metrics Ltd.) with six cameras situated $1.5-2 \mathrm{~m}$ from the subject. Data were sampled at 120 $\mathrm{Hz}$ and simultaneous inputs from the cameras were automatically converted into three-dimensional coordinates using Vicon software. Fifteen reflective markers (25 mm diameter) were placed over surface landmarks to monitor the motion of each body segment.

\subsection{Data Analysis}

The cross-correlation coefficient function $\rho(\tau)$ of two quantities $\mathrm{x}(\mathrm{t})$ and $\mathrm{y}(\mathrm{t})$ is the ratio of the cross-correlation function $R_{x y}(\tau)$ to the square root of the product of the autocorrelation functions of the two quantities at $\tau=0$ :

$\rho(\tau)=\frac{R_{x y}(\tau)}{\sqrt{R_{x z}(0) R_{y y}(0)}}$

For all $\tau$, the quantity $\rho(\tau)$ satisfies $-1 \leq \rho(\tau) \leq 1$. The function $\rho(\tau)$ measures the degree of linear dependence between $\mathrm{x}(\mathrm{t})$ and $\mathrm{y}(\mathrm{t})$ for a displacement of $\tau$ in $\mathrm{y}(\mathrm{t})$ relative to $\mathrm{x}(\mathrm{t})$. The correlation coefficient, symbolized by the $\mathrm{R}$ that ranges in value from $R=1$ for a perfect positive correlation to $R=-1$ for a perfect negative correlation. The coefficient of determination, symbolized as $R^{2}$, is simply the square of the correlation coefficient. The 
coefficient of determination can have only positive values ranging from $R^{2}=1$ for a perfect correlation (positive or negative) down to $R^{2}=0$ for a complete absence of correlation [11].

The coherence function between the input $\mathrm{x}(\mathrm{t})$ and the output $\mathrm{y}(\mathrm{t})$ of the experimental results is defined by

$$
r^{2}(f)=\frac{\left|G_{x y}(f)\right|^{2}}{G_{x x}(f) G_{v y}(f)}
$$

where $G_{x x}(f)$ and $G_{y y}(f)$ denote the autospectral density function of input and output, respectively, and $G_{x y}(f)$ is cross-spectral density function. The coherence function provides a formal measure of the correlation between two signals in the frequency domain. In the ideal case of a constant-parameter linear system with a single clearly defined input and output, the coherence function will be unity. If $\mathrm{x}(\mathrm{t})$ and $\mathrm{y}(\mathrm{t})$ are completely unrelated, the coherence function will be zero. The coherence function of 1 indicates that the phase shift between the waveform at a given frequency is constant, and the amplitude of the signals at that frequency has a constant ratio [11]. The coherence function can be interpreted as a measure of linear predictability [12-13] - it equals 1 whenever $\mathrm{x}(\mathrm{t})$ is a linear function of $\mathrm{y}(\mathrm{t})$, and it is also used in the calculation of $95 \%$ confidence limits on the transfer function data [14].

\section{Results and discussion}

\subsection{The reaction of the body to the platform translation}

The typical time course of the displacement with different vibration is shown in Fig. 2, for one
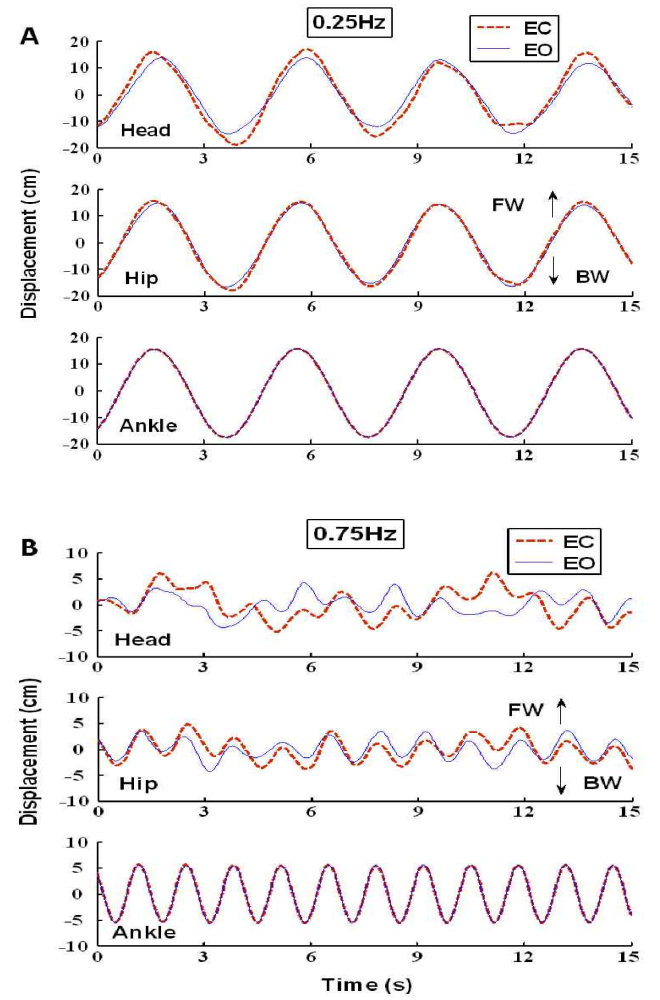

Fig. 2. Time course displacement of each body segment in the sagittal plane; Input: (A) $0.25 \mathrm{~Hz}$, (B) $0.75 \mathrm{~Hz}$; eyes closed (EC), eyes open (EO); forward (FW), backward (BW)

representative subject. This figure provide the displacement of each body segment, when the subject is exposed to sinusoidal $0.25 \mathrm{~Hz}$ perturbation with eyes open (EO) and eyes closed (EC) at a frequency range from $0.1 \mathrm{~Hz}$ to $1 \mathrm{~Hz}$ (Fig. 2A). The sinusoidal $0.75 \mathrm{~Hz}$ horizontal translation also has the same visual condition (Fig. 2B).

Each graph indicates the response of the three body segments (ankle, hip and head) in the sagittal plane; $0.25 \mathrm{~Hz}$ and $0.75 \mathrm{~Hz}$ sinusoidal horizontal translation; ankle (bottom trace), hip (middle), and head (top). The solid line indicates the response of each body segment with EO, and the dashed line follows EC.

The displacement of vertical direction in each 
graph points to the forward (FW) and backward (BW) direction. Note that the displacement of the designed stroke in $0.25 \mathrm{~Hz}$ (mean stroke: $0.1 \mathrm{~m}$ ) sinusoidal vibration is somewhat larger than that of $0.75 \mathrm{~Hz}$ (mean stroke: $0.03 \mathrm{~m}$ ). This is due to the low frequency sinusoidal movement of the supporting platform, which needs larger displacement for generating more severe postural perturbation. The displacement of the ankle remained equal to the trace of the platform in all trials, making it perfectly mirrored in the trace of the platform itself, as given that no relative movement of the foot with respect to the platform occurred. The peak-to-peak displacement of hip and head varied, however, depending on both the visual series and the type of vibration. A progressive amplification from the perturbation effect was present between $\mathrm{EO}$ and $\mathrm{EC}$.
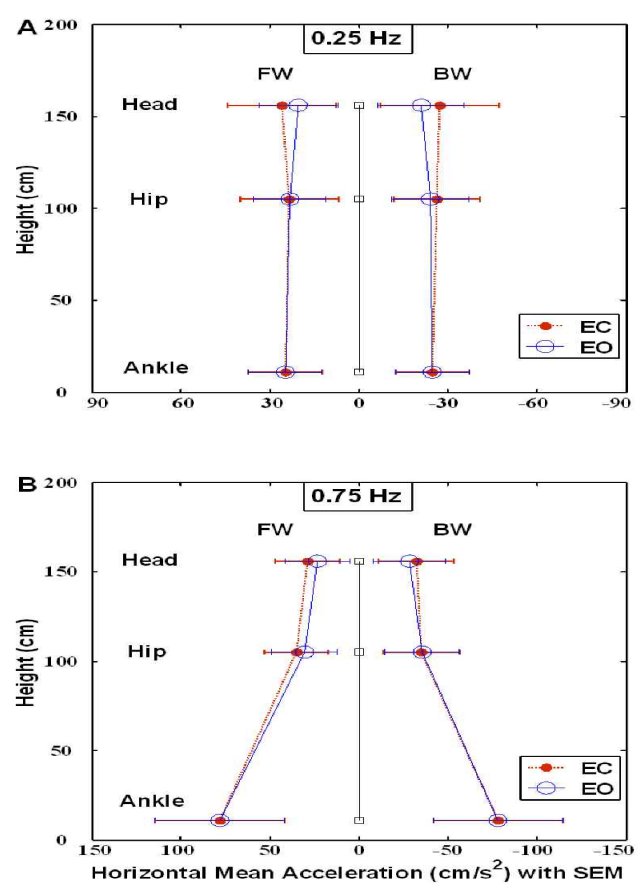

Fig. 3. Antero-posterior mean acceleration data with SEM of the markers positioned at ankle, hip, head under eyes open (EO) and eyes closed (EC)
Mean acceleration traces with SEM (Fig. 3) were derived from the displacement data in Fig. 2. It is averaged across all subjects of each trial. It shows schematically the average acceleration of the different body segments during each trial, under EO and EC, for both translation series (platform mean acceleration : $0.25 \mathrm{~m} / \mathrm{s}^{2}$ with $0.25 \mathrm{~Hz}, 0.78 \mathrm{~m} / \mathrm{s}^{2}$ with $0.75 \mathrm{~Hz}$ ).

Clearly, (1) In $0.75 \mathrm{~Hz}$ perturbation, since the subject was exposed to a relatively higher perturbation, its intermediate frequencies were not easily perceived to the subject and they were unable to adapt their standing posture to the unexpectedly changing profile of platform, anticipating it was also difficult; (2) though predictable, however, the subject can stand in a relatively stable at $0.25 \mathrm{~Hz}$ continuous periodic platform oscillation; (3) on average, the acceleration of hip and head was slighter than that of the ankle in $\mathrm{EO}$, while it varied to an increase in $\mathrm{EC}$; (4) the oscillation of head decreased at the higher frequency of periodic input and was particularly found when visual conditions were changed; (5) the acceleration of the head was larger in the backward direction than forward especially with EC, even though the subject has a large sized foot support base in front rather than behind the ankle, while the hip remained relatively around the limits of the platform excursion, even at the $0.75 \mathrm{~Hz}$ translation. In summary, the body was thrust beyond the limits of platform excursion with $\mathrm{EC}$, while head displacement minimized with EO. Correspondingly, since the hip motion also causes relatively large head motion, this may effects vestibular sensors within the skull, which may have implications on sensory feedback to the nervous system $[2,7-8]$.

\subsection{Cross-Correlation Analysis}

To understand the functional behavior of each 

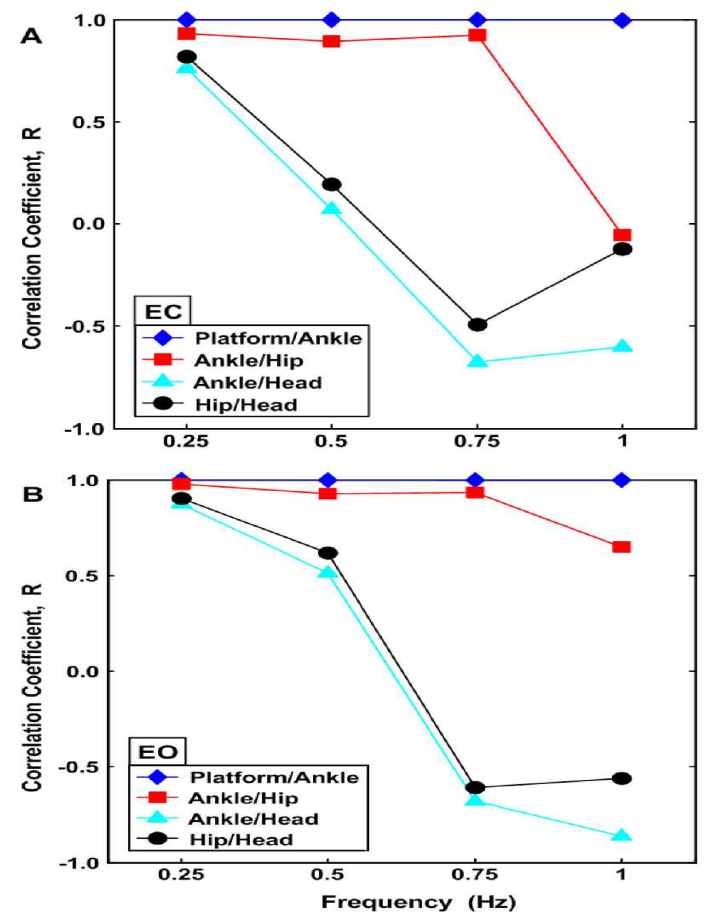

Fig. 4. Traces of the mean cross-correlation value between different body segments ( $A: E C$, $\mathrm{B}: \mathrm{EO})$

body segments under the horizontal perturbation, cross- $^{-}$correlation (CC) analysis has performed. This was calculated from acceleration data. Fig. 4 shows the correlation coefficient, $R$, and Fig. 5 shows the coefficient of determination, $R^{2}$. These CC profiles for the four pairs of the body segments (platform/ankle, ankle/hip, ankle/head, and hip/head) were averaged across all subjects for both visual series and type of translation.

There was a significantly high $R$ value $(>0.98)$ in the pair platform/ankle during all trials; not only a variation of visual series (EO and $\mathrm{EC}$ ) but also the high and low frequencies platform input, almost closed to 1 with positive direction. This value shows that the displacement of the ankle is always equal throughout the whole period of the platform motion [10-11].
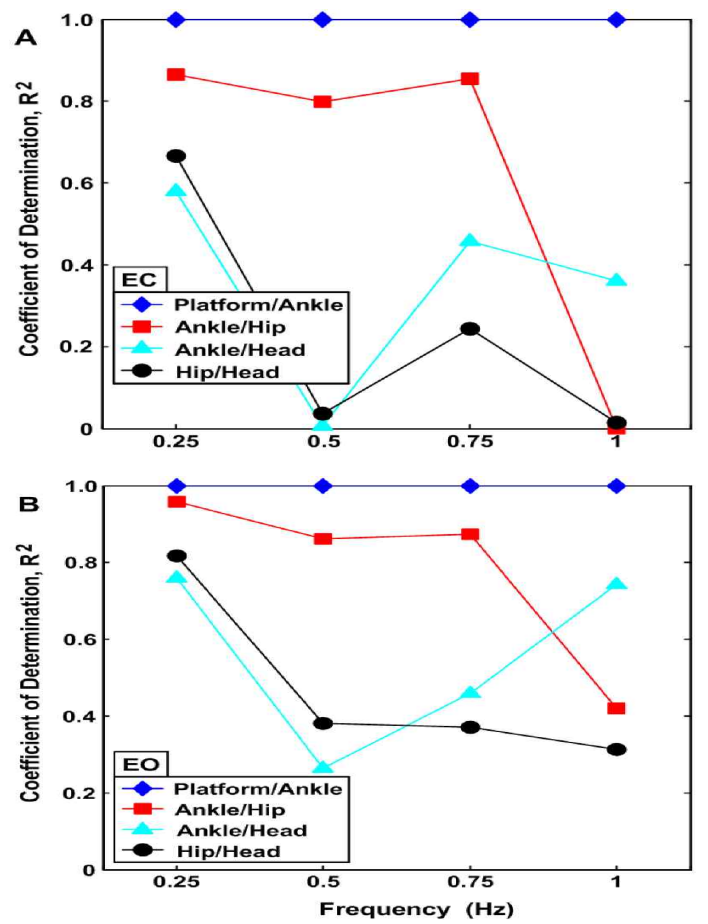

Fig. 5. Traces of the mean coefficient of determination value between different body segments ( $A: E C, B: E O)$

There were quite positive high $R$ values $(>0.76)$ for all body pairs at $0.25 \mathrm{~Hz}$ of platform input under both visual conditions. This indicates that all three body segments were moving almost concurrently at $0.25 \mathrm{~Hz}$ of platform input. This result shifted to low and negative values with ankle/head and hip/head at high translation frequency, while ankle/hip traces show positive high values (>0.89) up to $0.75 \mathrm{~Hz}$ under both visual conditions. Across the abscissa of Fig. 4, i.e., under the variation of platform input frequencies, these pairs show moderate traces with both visual conditions. Though it oscillated under different conditions of each trial, there were relatively negative high $R$ values in the pair ankle/head at $0.75 \mathrm{~Hz}(<-0.67$ in both $\mathrm{EC}$ and $\mathrm{EO})$, and $1.0 \mathrm{~Hz}(<-0.60$ with $\mathrm{EC},<-0.86$ with $\mathrm{EO})$. These $R$ values indicate that the ankle/head pair 
shows opposite direction of response to those platform inputs [10-11].

From the coefficient of determination, $R^{2}$ (see Fig. 5), the strength of the correlation between each body segment, in response to horizontal perturbation, can be estimated effectively. Though it varied, the ankle/head and hip/head pairs were present at lower $R^{2}$ values with frequency increase. On the contrary, the ankle/hip pair was much less affected by an increase in frequency of platform perturbation. The hip/head pair showed relatively low values at $0.5,0.75$, and $1 \mathrm{~Hz}$ platform translation among different body segment pairs under both visual conditions; in the $\mathrm{EC}$ trials particularly, it approached zero when subjects were exposed to 0.5 $\mathrm{Hz}$ and $1.0 \mathrm{~Hz}$ perturbations. This value was calculated at less than $R^{2}=0.03$. The $R^{2}$ values of the ankle/hip pair showed quite high values across all platform inputs under both visual conditions, except for $1 \mathrm{~Hz}$ ( $<0.01$ with $\mathrm{EC},<0.42$ with $\mathrm{EO})$.

\subsection{Functional Interpretation in Frequency Domain}

Since this study was dealing with various and continuous horizontal platform translation, the subject data signals were analyzed in both the time and frequency domain. The correlations between different body segments (ankle/hip, ankle/head, and hip/head) in the frequency domain, that is, coherence, are presented in Fig. 6.

These values were calculated from acceleration data for the four pairs of body segments (platform/ ankle, ankle/hip, ankle/head, and hip/head), and were averaged across all subjects for both visual series and type of translation. As expected, the position of the maximum coherence varied with the frequency band of the different pairs and the type of continuous horizontal translations.
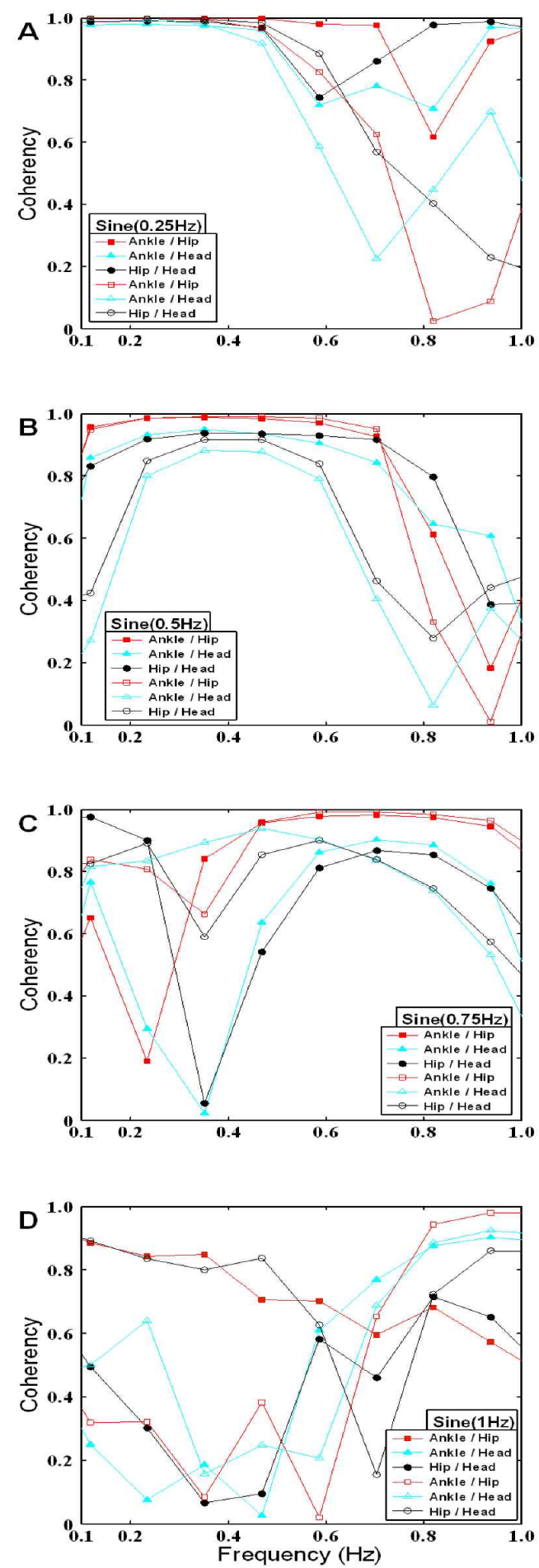

Fig. 6. Mean coherence functions analyses of the each different body segments $(A: 0.25 \mathrm{~Hz}$, $B: 0.5 \mathrm{~Hz}, C: 0.75 \mathrm{~Hz}, D: 0.1 \mathrm{~Hz} ; \mathrm{EC}:$ filled symbols, EO: open symbols) 
At the $0.25 \mathrm{~Hz}$ translation (Fig. 6A), high degrees of coherence $(>0.91)$ were found from $0.1 \mathrm{~Hz}$ to 0.45 $\mathrm{Hz}$ with all body segment pairs under both visual conditions. These results show that the internal reactions of each body segment were shown almost concurrent against external platform perturbation. Under the increment of platform input frequencies from $0.45 \mathrm{~Hz}$, it varied to show the individual radical variations. There were high coherence values from $0.23 \mathrm{~Hz}$ to $0.57 \mathrm{~Hz}$ at the $0.5 \mathrm{~Hz}$ platform input (Fig. $6 \mathrm{~B})$ with all body segment pairs under both visual conditions. These results showed a gradual decrease as the platform translation approached $1 \mathrm{~Hz}$, in particular where the lowest coherence values appeared, at $0.93 \mathrm{~Hz}$. This pattern indicates that there was more independent motion across all body pairs at $0.93 \mathrm{~Hz}$ platform perturbation [11-13]. The result was that $0.5 \mathrm{~Hz}$ frequency trials behaved as an intermediate pattern between $0.25 \mathrm{~Hz}$ and $0.75 \mathrm{~Hz}$ trials.

High coherence was displayed from $0.57 \mathrm{~Hz}$ to $0.82 \mathrm{~Hz}$ at the sinusoidal platform perturbation with $0.75 \mathrm{~Hz}$ (Fig. 6C), while individual radical variations were displayed from $0.1 \mathrm{~Hz}$ to $0.57 \mathrm{~Hz}$. This coherence value indicates that the internal reactions of body segments were displayed almost concurrently against external perturbation [14]. In these trials, the subjects easily adapted after a few cycles of platform translations since they needed minimal active efforts to maintain body equilibrium. Under these circumstances, the body kinetic energy is reduced and smaller. Moreover, even late active forces and the intrinsic stiffness of the body segments are possibly sufficient to maintain postural equilibrium to counteract the effect of the platform movement [2]. In comparison with $0.25 \mathrm{~Hz}$ platform input, the input perturbation of the platform was closer to stability limits at $0.75 \mathrm{~Hz}$ platform perturbation (Fig. 6C). This means that there are startle and strong inconsistent accelerative responses at more severe postural perturbation within the three pairs of body segments. Comparison between different trials under varying visual conditions also follows these patterns.

Though it drew closer to high coherence values as it approached $1 \mathrm{~Hz}$ frequency, the $1 \mathrm{~Hz}$ platform translation results (Fig. 6D) show that there were quite independent frequency responses across all body segment pairs at all frequency range, i.e., from $0.1 \mathrm{~Hz}$ to $1 \mathrm{~Hz}$. During the experiment, the subjects showed the most stable and comfortable postural behavior at $1 \mathrm{~Hz}$ input among different platform perturbations. These results can be possibly estimated from the effect of predictive platform movement. When subjects were exposed to relatively greater predictive perturbation, its intermediate frequencies were easily perceived by the subject and they were able to adapt their standing posture to the expected changing profile of platform, facilitating anticipation [3-4, 7]. Correspondingly, the equilibrium of each body segment was not severely challenged. Their standing postural mechanisms were possibly responsible for this, including trunk stabilization that can keep pace with the rapid, continuous inversion of the direction of the supporting platform.

\section{Conclusion}

The functional behavior of each body segment in standing postural dynamics was investigated in response to the horizontal translation in the sagittal plane. When stable human upright standing was disturbed by relatively non-predictive platform translation, its intermediate frequencies were not easily perceived by the subjects and they could not adapt their body balance to the irregularly changing profile of the platform and anticipation of changes 
was also difficult. When predictable, however, the subjects could stably withstand a condition of continuous easily predictive platform oscillating. The subjects' bodies were thrust beyond the limits of platform excursion without visual condition.

Ankle displacement was always equal to the trace of the platform in all trials, perfectly mirroring the translation of the platform itself. The cross-correlation result indicated that low relative movement exists between hip and head at relatively predictive platform translation under both visual conditions, while there was high significant relationship between ankle and hip, except at $1 \mathrm{~Hz}$. On average, the frequency domain analysis was consistent with the time domain results across all platform inputs especially when vision was allowed.

\section{References}

[1] L. M. Nashner, "Fixed patterns of rapid postural responses among leg muscles during stance," Exp. Brain Res., 1977.

[2] H. C. Diener, J. Dichgans, B. Guschlbauer, and H. Mau, "The significance of proprioception on postural stabilization as assessed by ischemia," Brain Res., 296: 103-109, 1984b.

[3] L. M. Nashner, "Adapting reflexes controlling the human posture," Exp Brain Res 26:59-72, 1976.

[4] F. B. Horak, H. C. Diener, and L. M. Nashner, "Influence of central set on human postural responses," J Neurophysiol 62:841-853, 1989.

[5] G. McCollum and T. K. Leen, "Form and exploration of mechanical stability limits in erect stance," J. Motor Behav., vol. 21, pp. 225-244, 1989.

[6] H. C. Diener, J. Dichgans, W. Bruzek, and H. Selinka, "Stabilization of human posture during induced oscillations of the body. Exp Brain Res 45:126-132, 1982.

[7] V. Dietz, M. Trippel, I. K. Lbrahim, and W. Berger, "Human stance on a sinusoidally translating platform: balance control by feedforward and feedback mechanisms,” Exp Brain Res 93:352-362, 1993.
[8] Tokita T, Myata H, and Fujiwara H, "Postural response induced by horizontal sway of a platform. Acta Otolaryngol Suppl," (Stockh) 406:120-124, 1984.

[9] Kuo A. D., Jajac F. E., "Human standing posture: multijoint movement strategies based on biomechanical constraints," Prog Brain Res, 97:349-358, 1993

[10] M. J. Griffin, "Handbook of human vibration," Academic press, 1996

[11] J. S. Bendat, and A. G. Piersol, "Measurement and analysis of random data," Wiley, New York, 1996.

[12] M. B. Priestley, Spectral Analysis and Time Series, Academic press, London, 1989.

[13] P. J. Brockwell and R. A. Davis, Time series: Theory and Methods, Springer, New York, 1991.

[14] R. K. Otnes and L. Enochson, Digital Time Series Analysis, Wiley, New York, 1972.

\section{저자소개}

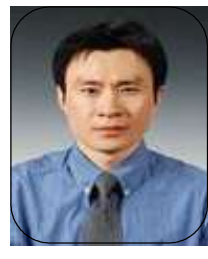

\section{Youngkyun Shin}

Youngkyun Shin received B.S. and M.S. degrees in Electrical and Computer Engineering from Kangwon National University, Korea in 1997 and 1999, respectively. He is currently an instructor at Yuhan and Inha Universities, and is pursuing a Ph.D. degree in the Graduate School of Information Sciences (GSIS), Tohoku University, Sendai, Japan. His research interests include design, control and applications of robotics, biomechanics, control of human posture and movement, and human-machine interaction.

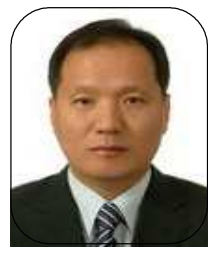

\section{Gu-Bum Park}

Gu-Bum Park received Bachelor, Master and Ph.D. degrees in Electrical Engineering from Inha University in 1984, 1986 and 1992, respectively. He joined the Department of Electrical Engineering, Yuhan University in 1992, where he is now a Professor. His current research interests include the fabrication of thin films as electrical devices, hard coatings and sensors. 